\title{
Editorial - Its Never Too Late: Healthy Lifestyles and Aging
}

Gerontologists are increasingly concerned with research questions that ask, To what extent do earlierlife events and experiences shape later-life outcomes, in particular, health status? And how do we promote healthy behaviours over the life course? Typically drawing from a number of interdisciplinary perspectives, such as continuity theory, developmental approaches, life-course theory, social change theory, and the transtheoretical model, researchers have demonstrated that healthy aging is not a static state but rather one that is the product of a multitude of interrelated and constantly changing life patterns. This conceptualization is also valid for advancing to healthy lifestyles, conceived of as patterns of behaviour - for instance, routine exercising, nutritious diet, and the absence of smoking and other "risk" behaviours, all of which are associated with positive health and wellbeing outcomes. Healthy lifestyles, in turn, are deemed to be a subset of the broader conception of lifestyle, defined by the World Health Organization as "a range of socially determined patterns of behaviour and interpretations of social situations, developed and used jointly by the group to cope with life" (1986, p. 118). In this sense, healthy lifestyles and the specific health behaviours comprising them are integrally tied to a person's life course, as well as to social status, social expectations, and health beliefs.

National, provincial, and local health strategies have adopted a population-health perspective that positions healthy lifestyle and the reduction of risk behaviours (e.g., obesity) at the heart of many policies and programs. However, the notion that healthy lifestyles are the product of life chances and choices accumulating over a person's life can have some serious, albeit unintended, connotations for persons at the top of the age pyramid. There appears to be an assumption that the greatest value added in our health-promoting efforts will be realized among persons who are younger, given that they reap the benefits of action for a longer period than do older persons. For example, if a young person quits smoking, there may be a more pronounced health benefit for the individual, in terms of reducing risk of illness, and for society, in terms of lessening the potential demand on the health care system. However, overemphasis on the targeting of health behaviours earlier in life can actually devalue and deter policies and action for lifestyle modification among persons reaching advanced ages. Extensive research has proven that the health benefits associated with improved lifestyle can accrue even if people are older adults. Yet there continues to be reluctance to put health promotion policies and programs into action in a way that meets the needs of an increasingly heterogeneous older population. Unquestionably, reversing stereotypes depicting the elderly as being relatively inactive and less prone to making alterations in lifestyle continues to be a barrier to realizing these aims in a predominantly youth-oriented society. Let us turn our attention to exercise, which is a behaviour that has been proven to reinforce a healthy lifestyle. Exercise will be used here interchangeably with physical activity, although it is understood that they have somewhat different definitions and operationalizations in the literature.

The lead article in this issue of the Canadian Journal on Aging, by Lalive d'Epinay and Bickel, entitled "Do 'Young-Old' Exercisers Feel Better Than Sedentary Persons? A Cohort Study in Switzerland", presents findings relevant to this discussion. The authors' study supports a causal link between sport/exercise trajectories measured between mid-life (close to age 50) and "young-old" (age 65 to 74) and well-being outcomes at these later ages. Four types of exercisers were defined: long-term exercisers (those continuing to exercise at least once a week between mid-life and becoming young-old), new exercisers (persons sedentary at mid-life who later become exercisers), quitters (those exercising at mid-life who stop by the time they are young-old), and sedentary (those persons who are sedentary both at mid-life and when young-old). Well-being measures include self-rated health and the Wang self-assessed depression scale. Specifically, they find that, as expected, long-term exercisers exhibit higher levels of well-being than quitters and sedentary persons. But even more intriguing is the finding that new exercisers rate their health at levels close to those of long-term exercisers, thus suggesting that individuals can make up for lost ground at least with respect to the social-psychological benefits of exercise. And finally, it is noteworthy that almost 40 per cent of their sample shifted, over that time period, from being exercisers to being non-exercisers and visa versa.

These results are consistent with an expansive research literature that has provided irrefutable evidence that an active lifestyle has positive benefits not only for physical but for subjective health, among persons of all ages, including older adults and the very old. They also strengthen the existence of a causal link between life-course exercise trajectories and health status. Furthermore, they reinforce the notion that exercise behaviour is not stable over the lives of indi- 
viduals. In fact, exercise patterns appear to be highly variable. This parallels other research demonstrating that exercise is influenced by reciprocal associations with health status change (and other characteristics) as we age. For example, an active lifestyle can delay the onset of illness, and conversely, illness or injury can act as a deterrent to exercise. Moreover, their research establishes that people who become exercisers between their 50 s and their late 60 s or 70 s enjoy many of the benefits inherent in that healthy lifestyle.

This begs the question: Are our health promotion efforts adequate to help people to initiate, sustain, or rekindle healthy lifestyles? On a positive note, the percentage of the population engaging in sedentary or infrequent levels of exercise has dropped significantly between the late 70s and now (Wister, 2003). Sedentary or infrequent exercise is arbitrarily defined in this research as less than three exercise intervals a week of 15 minutes or more in duration. This level is considered to be below the minimum recommended level and therefore is indicative of unhealthy lifestyle behaviour. Moreover, contrary to popular belief, this drop in unhealthy levels of exercise over the last 25 years has occurred at approximately the same rate among older persons as it has among adults of other ages. Yet, on a more negative note, the proportion of the population following minimum guidelines of basic healthy lifestyle practices is far from adequate from a population-health perspective. With respect to exercise levels, it is estimated that about 31 per cent of elderly women and 41 per cent of Canadians over 65 are moderate or better in their expenditure of energy, due to regular leisure-time physical activity, a level that is equated with a variety of health benefits (Health Reports, 1999). And exercise levels are not significantly better among the mid-life baby boom cohorts who will begin to turn 65 by 2011, or even in the population as a whole.

So, what is it that is keeping us from developing and sustaining active lifestyles across the life cycle? There is an enormous literature that addresses the causal mechanisms underlying physical activity patterns (e.g., see literature review by Sherwood and Jeffery, 2000). It is understood that physical activity is a complex, dynamic process that is influenced by a large constellation of individual, social, and environmental characteristics. These include both barriers (e.g., low self-efficacy) and facilitators (e.g., positive family support) to physical activity processes. What can be gleaned from this research is that a multipronged approach to active living that recognizes the heterogeneity of people of all ages is probably going to produce the greatest population-health improvements. This approach would entail policies and programs that cut across contexts linked to individuals, social groups, and society.

This necessitates expansion and further development of targeted exercise programs that meet the needs, resources, and readiness to change of individuals at various points in their life course, with different health conditions and support systems, and of different social classes and ethnicities. Turning to older adults, structured exercise programs should anticipate a certain degree of drop-out and improve methods of recruiting and maintaining participants who are experiencing a range of aging-related changes or illness symptoms. These activity programs need to be enjoyable, appear attractive to individuals with different tastes and preferences, and amalgamate social components to reinforce commitment and continued involvement. In addition, health professionals, especially general practitioners and family doctors, can play a more active role in supporting the adoption or continuation of healthy lifestyles of all kinds, given their influential position in society. And they can assist individuals in understanding best practice guidelines pertaining to exercise, diet, and medication interactions.

At a broader level, there is little doubt that societal attitudes need to change. Efforts to raise levels of physical activity significantly, among persons of all ages, through national campaigns, need to recognize the necessity of making exercise part of a lifestyle that can be supported through young adulthood, mid-life, and into older adulthood. At a policy level, there needs to be greater commitment by governments to make a physically active and healthy society a top priority. The construction and organization of safe bikeways and walkways, green-space, and parks are examples of ways that the built environment can be manipulated in a way to facilitate active lifestyles. In sum, it is imperative to propagate a powerful positive message that informs and teaches individuals that it is never too late to adopt or renew a healthy lifestyle.

Andrew V. Wister

Gerontology Programs

Simon Fraser University

\section{References}

Health Reports. (1999). Personal health practices: Smoking, drinking, physical activity and weight. Health Reports, 11(3), 83-90.

Sherwood, N., \& Jeffery, R. (2000). The behavioural determinants of exercise: Implications for physical activity interventions. Annual Review of Nutrition, 20, 21-44.

Wister, A.V. (2003). Baby boomer health dynamics: How are we aging? Unpublished manuscript.

World Health Organization. (1986). Lifestyles and health. Social Science and Medicine, 22, 117-124. 